\title{
Enhanced Second Harmonic Generation in Femtosecond Laser Inscribed Double-Cladding Waveguide of Nd:GdCOB Crystal
}

\author{
Yuechen Jia, J. R. Vázquez de Aldana, Qingming Lu, Daniel Jaque, and Feng Chen
}

\begin{abstract}
We report on the fabrication of double-cladding waveguides in Nd:GdCOB crystals by using femtosecond laser inscription with scanning speed of $0.5 \mathrm{~mm} / \mathrm{s}$. This prototype consists of two concentric tubular structures with nearly circular cross sections with different diameters. The fabricated cladding waveguides possess relatively low propagation losses of less than $0.65 \mathrm{~dB} / \mathrm{cm}$. The micro photoluminescence and second harmonic confocal images of the fabricated waveguides, which were performed by using a confocal microscope, have revealed that neither the fluorescence nor the nonlinear properties of the constituent crystals have been deteriorated during femtosecond laser micromachining procedure. Under the pulsed laser pumping at fundamental wavelength of $1064 \mathrm{~nm}$, the guided wave second harmonic generation (SHG) at $532 \mathrm{~nm}$ has been realized from the single and double cladding waveguides. Compared to the single-claddings $(2.3$ or $4.7 \%$, depending on the diverse cladding diameters), the double cladding architecture has been found to be with enhanced SHG conversion efficiency $(\sim 5.1 \%)$.
\end{abstract}

Index Terms-Femtosecond laser inscription, Nd:GdCOB crystal, optical waveguides, second harmonic generation (SHG).

\section{INTRODUCTION}

A S ONE of the most promising candidate for selffrequency-doubling (SFD) lasers, the implementation of laser diode directly pumped visible lasers by employing neodymium-doped gadolinium calcium oxoborate $\left(\mathrm{Nd}: \mathrm{GdCa}_{4} \mathrm{O}\left(\mathrm{BO}_{3}\right)_{3}\right.$ or $\left.\mathrm{Nd}: \mathrm{GdCOB}\right)$ crystals is of intriguing

Manuscript received September 1, 2013; revised October 25, 2013; accepted October 26, 2013. Date of publication November 3, 2013; date of current version November 20, 2013. The work was supported by the National Natural Science Foundation of China under Grants 11274203 and 11111130200), the Spanish Ministerio de Ciencia e Innovación (MICINN) through Consolider Program SAUUL CSD2007-00013 and Project FIS2009-09522, and the Centro de Láseres Pulsados (CLPU).

Y. Jia and F. Chen are with the School of Physics, and State Key Laboratory of Crystal Materials, Shandong University, Jinan 250100, China (e-mail: jiayuechen0521@163.com; drfchen@sdu.edu.cn).

J. R. Vázquez de Aldana is with the Laser Microprocessing Group, Departamento de Física Aplicada, Universidad de Salamanca, Salamanca 37008, Spain (e-mail: -jrval@usal.es).

Q. Lu is with the School of Chemistry and Chemical Engineering, Shandong University, Jinan 250100, China (e-mail: qmlu@ sdu.edu.cn).

D. Jaque is with the Fluorescence Imaging Group, Departamento de Física de Materiales, Facultad de Cienicas, Universided Autónoma de Madrid, Madrid 28049, Spain (e-mail: daniel.jaque@uam.es).

Color versions of one or more of the figures in this paper are available online at http://ieeexplore.ieee.org.

Digital Object Identifier 10.1109/JLT.2013.2288371 interest owing to its outstanding photoluminescence features as well as to the nonlinear properties of GdCOB matrix [1]-[4]. Waveguide geometry could confine and control light propagation within extremely compressed volumes with dimensions of micrometer or submicrometer scales. Due to this fact, optical intensities in guiding structures could reach a much higher level compared to the bulk [5], [6]. Consequently, a series of considerably enhanced performances correlated to the substrate materials could be realized in the waveguide configurations, benefiting from the larger optical intensities, such as low pump threshold for waveguide lasers, fast response for photorefractive waveguides as well as multiple configurations for frequency conversion for non-linear waveguides [7]-[9]. Particularly, based on the SFD crystals, it is possible to facilitate the integration of the intracavity visible waveguide lasers that do not require the additional frequency conversion through a nonlinear crystal waveguide [10]. Thus, Nd:GdCOB waveguides could be a suitable platform to host the intracavity for the laser generation of infrared light and synchronously realize the nonlinear process in the same structure. Nevertheless, the first significant step to realize the SFD waveguide lasers is to obtain the second harmonic generation (SHG) in nonlinear waveguides, e.g., $1064 \rightarrow 532 \mathrm{~nm}$ optical conversion.

Since the pioneering work of Davis et al. in 1996 [11], femtosecond (fs) laser inscription technique has been emerged to be a powerful and efficient method for the construction of threedimensional guiding structures inside numerous transparent materials, and a wide range of photonic applications have been realized [12]-[25]. These guiding structures include the single-line written waveguides (so-called Type I, with positive refractive index changes in the irradiated filament) [13], double-line fabricated waveguides (so-called Type II, with stress-induced guiding region between the two tracks of negative index changes) [14]-[18], Type III depressed cladding waveguides (located in the core surrounded by multiple low-index tracks) [19]-[25]. Compared to single- and double-line fabricated waveguides, one of the advantages of the normal cladding waveguiding structures is that the large scale cross sections could match the commercially available multimode fibers (with diameters of $100-400 \mu \mathrm{m}$ ), which in principle offers an opportunity to realize efficient fiber-waveguide laser systems with low costs. More recently, a novel configuration consists of two concentric tubular structures with nearly circular cross sections with different diameters, so called double-cladding waveguides, has 


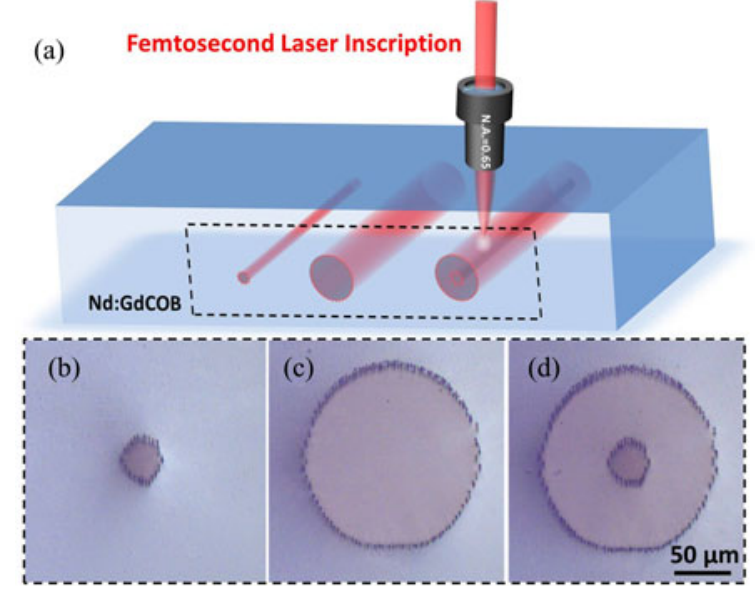

Fig. 1. (Color Online) (a) Fabrication schematic of fs-laser inscription process in Nd:GdCOB crystals, and optical images of the single- (with diameters of (b) 30 and (c) $150 \mu \mathrm{m}$ ) as well as (d) double-cladding waveguides.

been constructed in Yb:YAG ceramics [23], Nd:YAG ceramics [24] and Nd:YAG crystals [25]. With this configuration, the lasing performance of the waveguide was significantly enhanced with respect to standard single cladding waveguide, benefiting from the large pump area of the outer-most cladding [23]-[25]. In this paper, we report on the fabrication of double-cladding waveguides in Nd:GdCOB crystals by using fs laser inscription. The micro-photoluminescence ( $\mu$-PL), and micro-second harmonics $(\mu-\mathrm{SH})$ experiments have been performed to determine and localize the micro-structural changes induced in the Nd:GdCOB network, to elucidate the possible refractive index mechanisms leading to waveguiding. Comparing to the standard single cladding waveguide, the double-cladding structure was found to show superior SHG performances, with enhanced conversion efficiency and larger second harmonic (SH) output power, which suggests the potential applications of this double-cladding waveguides as multi-functional integrated laser sources. This is to our best knowledge for the first time the SHG enhancement of fs-laser inscribed double-cladding waveguides.

\section{EXPERIMENTS IN DETAILS}

The optically polished Nd:GdCOB single crystal sample used in this work were cut into wafers with dimensions of $10 \times 8 \times$ $2 \mathrm{~mm}^{3}$ to fit the direction of the $1064 \rightarrow 532 \mathrm{~nm}$ birefriengent PM SHG in the $x-z$ plane (i.e., $\theta=161.5^{\circ}, \varphi=0^{\circ}$ ). The single and double depressed cladding guiding structures were fabricated in Nd:GdCOB sample by utilizing the laser facilities at the Universidad de Salamanca, Spain, as schematized in Fig. 1(a). We used an amplified Ti:Sapphire laser system (Spitfire, Spectra Physics, USA) generating linearly-polarized $120 \mathrm{fs}$ pulses at a central wavelength of $800 \mathrm{~nm}$ (with $1 \mathrm{kHz}$ repetition rate and $1 \mathrm{~mJ}$ maximum pulse energy). The value of the pulse energy used to irradiate the sample was set with a calibrated neutral density filter, a half-wave plate and a linear polarizer. The sample was placed in a computer controlled motorized 3-axes stage. The beam was focused through a $40 \times$ microscope objective (N.A. $=0.65$ ) at certain depth beneath the largest sample surface (dimensions of $10 \times 8 \mathrm{~mm}^{2}$ ), and several tests at different pulse energies and scanning velocities were performed. Optical microscopy (in transmission mode) was used to evaluate the damage tracks produced in the sample and the final irradiation parameters were fixed to $0.84 \mu \mathrm{J}$ of pulse energy. During the irradiation the sample was moved at a constant speed of $500 \mu \mathrm{m} / \mathrm{s}$ in the direction perpendicular to the laser polarization and the pulse propagation that was carefully aligned with the $10-\mathrm{mm}$ long edge of the sample, thus producing a damage track along the sample. Such values were chosen as a compromise between producing a large enough damage in the laser tracks and minimizing the formation of cracks in the sample. Many parallel scans (with $\sim 3 \mu \mathrm{m}$ separation between adjacent damage tracks) were done at different depths of the sample (from bottom to top in order to avoid the shielding of the incident pulses by the previously written damage tracks) to inscribe the single- and double-cladding waveguides. The combination of a tubular central structure with $30 \mu \mathrm{m}$ diameter [the same size to the $30 \mu \mathrm{m}$ diameter cladding structure, as shown in Fig. 1(b)] and a concentric larger size tubular cladding with $150 \mu \mathrm{m}$ diameter [the same condition to the $150 \mu \mathrm{m}$ singlecladding waveguide in Fig. 1(c)] consist the double-cladding configuration, see Fig. 1(d).

The contrast of the refractive index of the tracks and waveguide was estimated to be $\Delta n \approx 0.008$ by measurement of the N.A. of the waveguides using the equation of

$$
\Delta n \approx \frac{\sin ^{2} \Theta_{\mathrm{m}}}{2 n}
$$

where $n=1.7180$ is the substrate refractive index at $632.8 \mathrm{~nm}$, $\Theta_{m} \approx 9.5^{\circ}$ is the maximum beam divergence of the light entering or leaving the waveguide [18]. In addition, we also performed the waveguide losses measurements at $632.8 \mathrm{~nm}$, taking the Fresnel reflection loss of the end facets (in our case, the value is about $0.3 \mathrm{~dB}$ ) into account. The insertion losses of the $150 \mu \mathrm{m}$ single-cladding structure was estimated to be $0.5 \mathrm{~dB} / \mathrm{cm}$, while, for the $30 \mu \mathrm{m}$ single-cladding and $30 \mu \mathrm{m}$ inner core of doublecladding waveguides, the values were both around $0.65 \mathrm{~dB} / \mathrm{cm}$. The higher attenuation for the cladding waveguides should be partly attribute to the damaged filaments at the waveguide contour fabricated by fs micromachining, which brings out the additional attenuation through the scattering. And the more compact the guiding structures are, the damaged filaments would affect the light propagation more obvious.

In order to study the nature and spatial location of these fs-modified volumes as well as to evaluate the potential application of the obtained waveguides as laser gain medium, $\mu$-PL and $\mu$-SH measurements have been performed by utilizing an Olympus BX-41 fibre-coupled confocal microscope at the Universidad Autónoma de Madrid, Spain. A mode-locked Ti:Sapphire laser (Millenia Spectra Physics) was employed to deliver $100 \mathrm{fs}$ pulsed laser beam, linearly polarized at $800 \mathrm{~nm}$ and with a repetition rate of $80 \mathrm{MHz}$, which is focused into the sample by using a $100 \times$ microscope objective with a N.A. of 0.85 . The subsequent backscattered radiation signals ( $\mu$-PL and $\mu$-SH, depending on the end face under study) were collected with the same objective and, after passing through a set of 


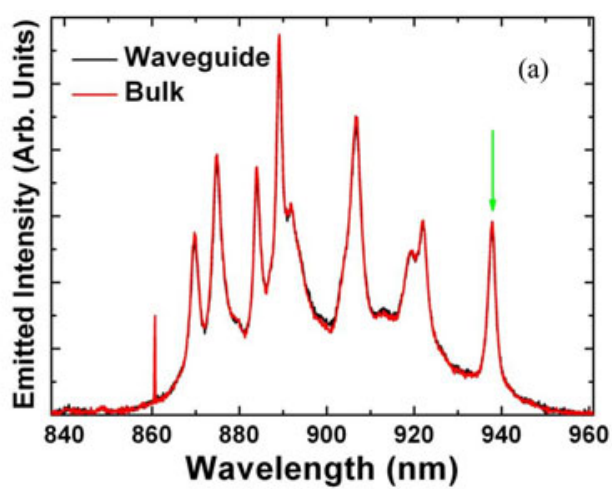

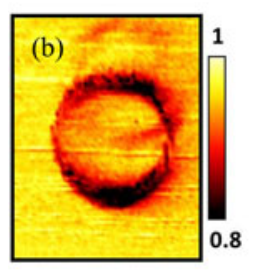

Intensity

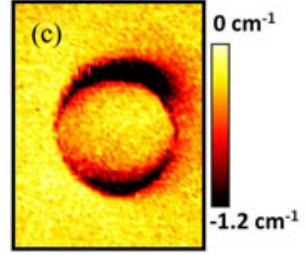

Shift

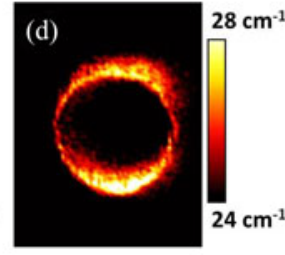

Width
Fig. 2. (Color Online) (a) Room-temperature fluorescence emission spectra $\left(\mu\right.$-PL) of $\mathrm{Nd}^{3+}$ ions related to the ${ }^{4} \mathrm{~F}_{3 / 2} \rightarrow{ }^{4} \mathrm{I}_{9 / 2}$ band as obtained from the fs-inscribed Nd:GdCOB cladding waveguide (the $150 \mu$ m diameter singlecladding waveguide) and bulk. And two-dimensional spatial distribution of the (b) emitted intensity, (c) energy shift and (d) FWHM of the $\mathrm{Nd}^{3+}$ emission lines obtained from the end face of the same waveguide.

filters, lenses and pinholes, it was spectrally analyzed by a high resolution spectrometer. The sample was mounted on an $x-y$ motorized stage with the spatial resolution of $100 \mathrm{~nm}$.

We used a typical end-face coupling system to characterize the nonlinear performances of the fs-fabricated waveguides. For the SHG experiments of Nd:GdCOB single- and double-cladding waveguides, a pulsed laser beam at a wavelength of $1064 \mathrm{~nm}$ as the fundamental wave, was coupled into the waveguides by a convex lens with focal length of $f=25 \mathrm{~mm}$. The laser produced $80 \mu \mathrm{J}$ pulses with a duration of $11.05 \mathrm{~ns}$ at a repetition of $5 \mathrm{kHz}$, with a maximum average power of $480 \mathrm{~mW}$. The light emerging from the output facet of the waveguides was captured by employing a microscope objective lens with a N.A. of 0.4 . And the generated SH light was separated from the leaked fundamental laser beam by utilizing a mirror with transmission of $\sim 70 \%$ at $\sim 532 \mathrm{~nm}$ and reflectivity $>99 \%$ at $\sim 1064 \mathrm{~nm}$, and then detected by a spectrometer, an infrared CCD camera and a powermeter.

\section{RESULTS AND DISCUSSION}

Fig. 2(a) depicts the room-temperature fluorescence emission spectra of $\mathrm{Nd}^{3+}$ ions related to the ${ }^{4} \mathrm{~F}_{3 / 2} \rightarrow{ }^{4} \mathrm{I}_{9 / 2}$ band as obtained from the waveguide (the $150 \mu \mathrm{m}$ single-cladding waveguide) and bulk. As we can see, the emission spectra are virtually identical indicating that the fluorescence efficiency of $\mathrm{Nd}^{3+}$ ions has been well preserved in the waveguide volume during the fs-laser inscription procedure, suggesting potential applications as integrated laser sources. Similar properties were found from the $30 \mu \mathrm{m}$ single-cladding and double-cladding waveg-
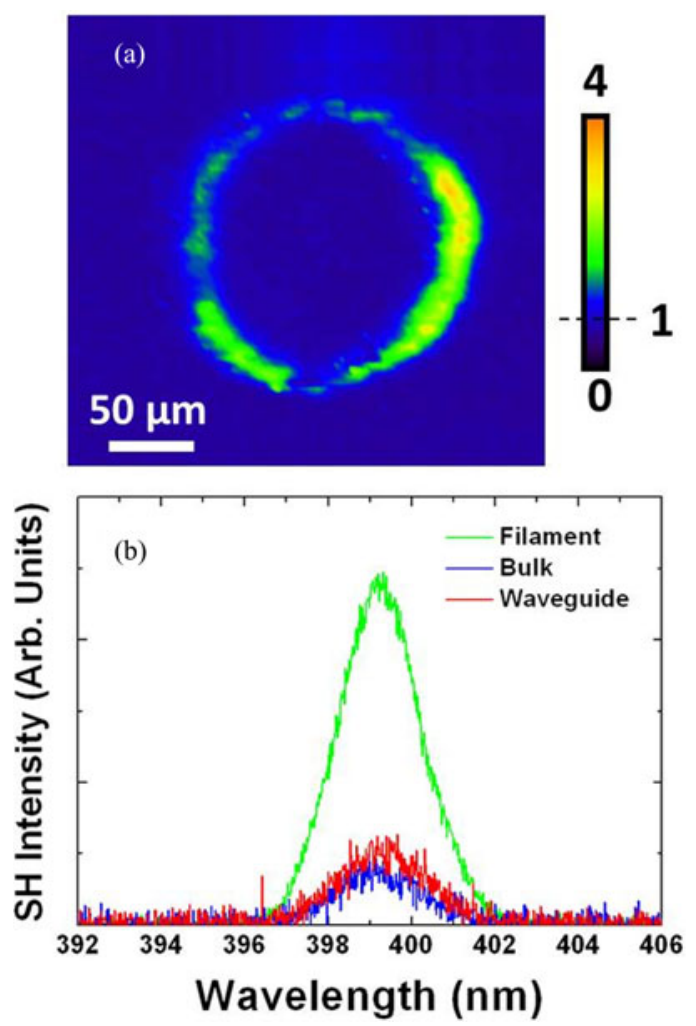

Fig. 3. (Color online) (a) Confocal $\mu$-SH cross-sectional image of cladding waveguide (the $150 \mu \mathrm{m}$ diameter single-cladding waveguide) fabricated in the $\mathrm{Nd}$ :GdCOB crystal as obtained from the end face. And the (b) emitted intensity spectra of $\mu$-SH when the 100 fs laser spot (at $800 \mathrm{~nm}$ ) is focused at three different positions (i.e., damage filaments, bulk and waveguide).

uides. A detailed analysis of the emission spectra reveals that slight changes in both the spectral position and width of the emission lines have been produced at the fs laser processed areas, which is clear evidenced in the fluorescence images obtained from the $150 \mu \mathrm{m}$ single-cladding waveguide, as included in Fig. 2(b)-(d) (corresponding to the spatial variation of the intensity, induced spectral shift and broadening of the $\mathrm{Nd}^{3+}$ fluorescence line at around $940 \mathrm{~nm}$ ). It is clear that the marked modifications of fluorescence intensity, spectral shift as well as fluorescence width only appear at the waveguide contour, where the $\mathrm{Nd}$ :GdCOB network has been simultaneously damaged, compressed and disordered. At the waveguide's volume, the Nd:GdCOB network has been only slightly modified, resulting in a partial dilatation that is not accompanied by any relevant disorder nor damage. This could be explained in terms of the high density of defects induced at the focus position of fs laser pulses due to the creation of a highly confined optical energy that leads to a well localized optical breakdown. It results in a permanent damage, which causes a low-index barrier that could confines light propagation within the structure. As commented previously, these maps also denote a good preservation of fluorescence efficiency at the waveguide's volume. In addition, as it can be observed from Fig. 3(a), the nonlinear response of the $\mathrm{Nd}: \mathrm{GdCOB}$ crystal has not been modified in the waveguide volume, but only at the waveguide contour instead (i.e., the locations of damage tracks). In Fig. 3(b) it is clear 


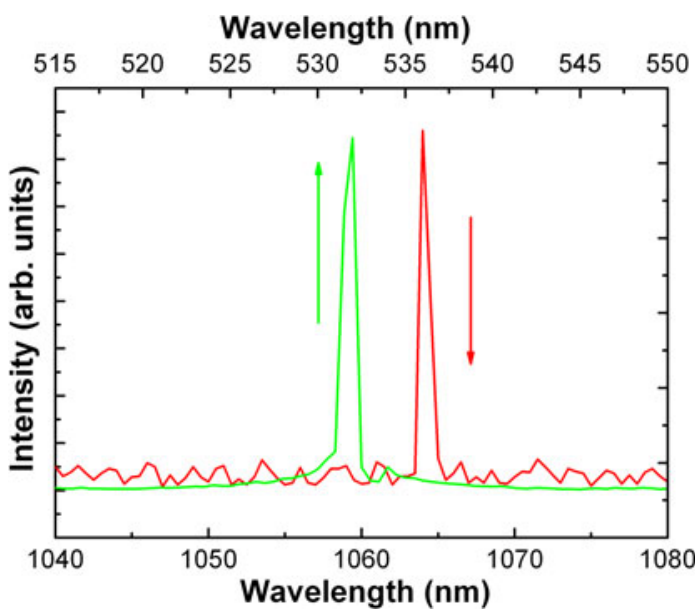

Fig. 4. (Color online) Laser spectra of the fundamental (red) and SH (green) waves from the $150 \mu \mathrm{m}$ diameter Nd:GdCOB single-cladding waveguide produced by fs-laser inscription.

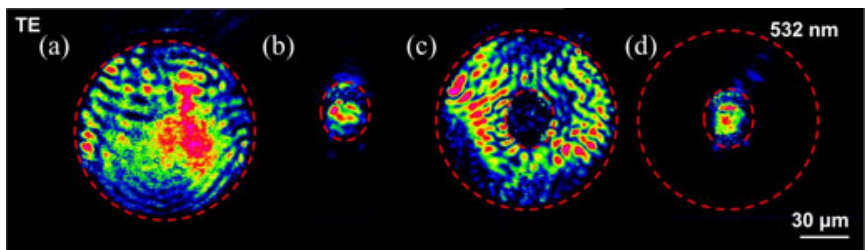

Fig. 5. (Color online) The modal profiles of $\mathrm{SH}$ waves at $532 \mathrm{~nm}$ from the (a) $150 \mu \mathrm{m}$, (b) $30 \mu \mathrm{m}$ diameter single-cladding waveguides and the (c) inner and (d) outer part of double-cladding waveguides. The red dashed lines indicate the spatial location of the waveguides.

evidenced that the generated SH intensity is very similar in bulk and inner volumes whereas. A clear $\mu$-SH enhancement (with respect to bulk) is found at the damage zones (about 4 times the emitted intensity of bulk material), which can be attributed to an enhancement in the back-reflection efficiency due to the presence of defects acting as scattering centers. Therefore, we can conclude from Figs. 2 and 3 that both the laser and nonlinear properties of the $\mathrm{Nd}$ :GdCOB network have not been deteriorated as a consequence of the waveguides fabrication procedure.

The typical laser spectrum of the fundamental (at $1064 \mathrm{~nm}$ ) and $\mathrm{SH}$ (at $532 \mathrm{~nm}$ ) waves from the $150 \mu \mathrm{m}$ diameter $\mathrm{Nd}$ :GdCOB single-cladding waveguide under the pulsed laser pumping is shown in Fig. 4, clearly depicts the nonlinear process of SHG in Nd:GdCOB waveguides. The fundamental wave at $1064 \mathrm{~nm}$ is at TM polarization and the SH wave is with TE polarization, i.e., the SHG process occurs under $\mathrm{TM}^{\omega} \rightarrow \mathrm{TE}^{2 \omega}$, which is in well agreement with the bulk case.

Fig. 5(a)-(d) depict the SH guided modal images from the $150,30 \mu \mathrm{m}$ single-cladding waveguides and the inner core, outer ring-shaped part of the double-cladding waveguide, respectively. As we can see, all the cladding structures support multi-mode waveguide lasers at $532 \mathrm{~nm}$ with TM polarization, which can be well understood due to the large scales of the structures. Interestingly, besides the modal distribution confined in the inner claddings [in Fig. 5(d)], the double-cladding waveguides also show ring-shaped modal profiles [Fig. 5(c)], which
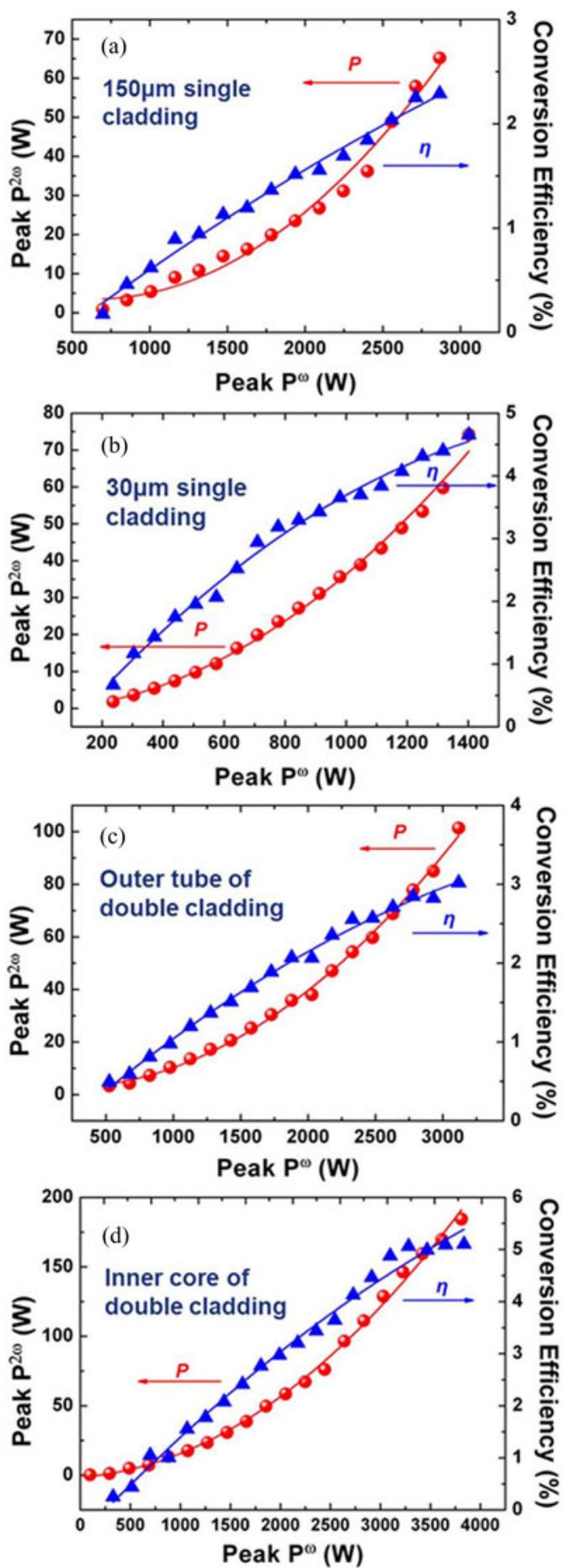

Fig. 6. (Color online) Output $\mathrm{SH}$ laser power at $532 \mathrm{~nm}$ as a function of fundamental pump power obtained from the Nd:GdCOB cladding waveguides in peak power: from the single-cladding with diameters of (a) 150 and (b) $30 \mu \mathrm{m}$, (c) in the ring-shaped regions and (d) inner core of $30 \mu \mathrm{m}$ diameter from the double-cladding waveguides.

are confined in the loop between the 150 and $30 \mu \mathrm{m}$ concentric tubes.

Fig. 6 depicts the generated SH wave powers (peak power) at $532 \mathrm{~nm}$ as a function of the absorbed fundamental pump power at $1064 \mathrm{~nm}$ from the Nd:GdCOB single- and double-cladding waveguides. The solid circles and the lines are the experimental 
data and the linear fit, respectively. As shown in this figure, the maximum output peak powers of the SH light are 65, 74, 101 and $184 \mathrm{~W}$ for the $150 \mu \mathrm{m}$ [Fig. 6(a)], $30 \mu \mathrm{m}$ [Fig. 6(b)] singlecladding waveguides, the outer ring-shaped section [Fig. 6(c)] and inner core [Fig. 6(d)] of double-cladding waveguides with conversion efficiencies of $2.3 \%, 4.7 \%, 3.0 \%$ and $5.1 \%$, respectively. By comparison, we can easily find that in both singleand double-cladding cases, the more compressed guiding structures (i.e., $30 \mu \mathrm{m}$ single-cladding and double-cladding core) show better SHG performances than those comparable incompact geometries (i.e., $150 \mu \mathrm{m}$ single-cladding and ring-shaped section of double-cladding) with considerable enhancement of maximum output powers as well as SHG conversion efficiencies. This phenomenon must be benefited from more compact guiding structures with enhanced spatial confinement of light fields, and in which higher optical intensities could be obtained. In addition, the inner core of double-cladding, which with the same size and shape to $30 \mu \mathrm{m}$ single-cladding waveguide, exhibits superior SHG performance. It should be due to the largearea pumping but compressed output volume, which means that more pumping fundamental power goes into the whole structure but only generates guided $\mathrm{SH}$ waves in the inner core region. This advantage of the double-cladding structures reflects on the SHG performances, resulting in increased output powers and enhanced conversion efficiency. It should be pointed out that even the ring-shaped section of double-claddings show superior SHG property to the $150 \mu \mathrm{m}$ single-cladding waveguides, which with the same diameter to the ring-shaped structure.

\section{CONCLUSION}

In conclusion, we have fabricated concentric tubular waveguides with double claddings in $\mathrm{Nd}$ :GdCOB crystal by using fs laser inscription. Confocal $\mu$-PL and $\mu$-SH images have revealed that both the fluorescence properties and the nonlinear response of the $\mathrm{Nd}$ :GdCOB crystal have been well preserved within the waveguide volume, which suggests the formed structures are promising candidates for the near-future development of efficient SFD laser waveguides operating in the visible. The SHG based on birefringent Type I phase matching from 1064 to $532 \mathrm{~nm}$ has been realized under pulsed laser configuration. The $\mathrm{SH}$ waves with conversion efficiency of $5.1 \%$ and maximum output peak power of $184 \mathrm{~W}$ from the inner core of doublecladding has shown superior SHG performance to the standard single-cladding waveguides, which is benefited from the largearea pump of outermost cladding. It should be pointed out that, since the large diameters of the outer claddings are comparable with those of the optical fibers, this paves a way to construct an integrated laser system with a direct fiber-waveguide configuration.

\section{REFERENCES}

[1] G. Aka, A. Kahn-Harari, D. Vivien, J. M. Benitez, F. Salin, and J. Godard, "A new nonlinear and neodymium laser self-frequency doubling crystal with congruent melting: $\mathrm{Ca}_{4} \mathrm{GdO}\left(\mathrm{BO}_{3}\right)_{3}$ (GdCOB)," Eur. J. Solid State Inorg. Chem., vol. 33, no. 8, pp. 727-736, 1996.

[2] G. Aka, A. Kahn-Harari, F. Mougel, D. Vivien, F. Salin, P. Coquelin, P. Colin, D. Pelenc, and J. P. Damelet, "Linear- and nonlinear-optical properties of a new gadolinium calcium oxoborate crystal,
$\mathrm{Ca}_{4} \mathrm{GdO}\left(\mathrm{BO}_{3}\right)_{3}$," J. Opt. Soc. Amer. B, vol. 14, no. 9, pp. 2238-2247, Sep. 1997.

[3] F. Mougel, G. Aka, A. Kahn-Harari, H. Hubert, J. M. Benitez, and D. Vivien, "Infrared laser performance and self-frequency doubling of $\mathrm{Nd}^{3+}: \mathrm{Ca}_{4} \mathrm{GdO}\left(\mathrm{BO}_{3}\right)_{3}$ (Nd:GdCOB)," Opt. Mater., vol. 8, no. 3, pp. $161-$ 173, Sep. 1997.

[4] C. Chen, T. Sasaki, R. Li, Y. Wu, Z. Lin, Y. Mori, Z. Hu, J. Wang, G. Aka, and M. Yoshimura, Nonlinear Optical Borate Crystals: Principals and Applications. Weinheim, Germany: Wiley, 2012.

[5] M. L. Calvo and V. Lakshminarayanan, Optical Wave-guides: From Theory to Applied Technologies. London, U.K.: CRC Press, 2007.

[6] G. Lifante, Integrated Photonics: Fundamentals. Chichester, U.K.: Wiley, 2008.

[7] C. Grivas, "Optically pumped planar waveguide lasers, Part I: Fundamentals and fabrication techniques," Progr. Quantum Electron., vol. 35, no. 6 , pp. 159-239, Nov. 2011.

[8] D. Kip, "Photorefractive waveguides in oxide crystals: Fabrication, properties, and applications," Appl. Phys. B, vol. 67, no. 2, pp. 131-150, May 1998.

[9] G. I. Stegeman and C. T. Seaton, "Nonlinear integrated optics," J. Appl. Phys., vol. 58, no. 12, pp. R57-R77, Sep. 1985.

[10] M. Fujiamura, T. Kodama, T. Suhara, and H. Nishihara, "Quasi-phasematched self-frequency-doubling waveguide laser in $\mathrm{Nd}: \mathrm{LiNbO}_{3}, "$ IEEE Photon. Technol. Lett., vol. 12, no. 11, pp. 1513-1515, Nov. 2000.

[11] K. M. Davis, K. Miura, N. Sugimoto, and K. Hirao, "Writing waveguides in glass with a femtosecond laser," Opt. Eng., vol. 21, pp. 1729-1731, 1996.

[12] F. Chen and J. R. Vázquez de Aldana, "Optical waveguides in crystalline dielectric materials produced by femtosecond-laser micromachining," Laser Photon. Rev., DOI: 10.1002/lpor.201300025, May 2013.

[13] J. Burghoff, S. Nolte, and A. Tünnermann, "Origins of waveguiding in femtosecond laser-structured $\mathrm{LiNbO}_{3}$," Appl. Phys. A, vol. 89, no. 1, pp. $127-132$, Oct. 2007.

[14] A. Ródenas, G. A. Torchia, G. Lifante, E. Cantelar, J. Lamela, F. Jaque, L. Roso, and D. Jaque, "Refractive index change mechanisms in femtosecond laser written ceramic Nd:YAG waveguides: micro-spectroscopy experiments and beam propagation calculations," Appl. Phys. B, vol. 95, no. 1, pp. 85-96, Apr. 2009.

[15] T. Calmano, J. Siebenmorgen, F. Reicher, M. Fechner, A. Paschke, N. Hansen, K. Petermann, and G. Huber, "Crystalline Pr:SrAl1 ${ }_{2} \mathrm{O}_{19}$ waveguide laser in the visible spectral region," Opt. Lett., vol. 36, no. 23, pp. 4620-4622, Dec. 2011.

[16] A. Ródenas, A. Benayas, J. R. Macdonald, J. Zhang, D. Y. Tang, D. Jaque, and A. K. Kar, "Direct laser writing of near-IR step-index buried channel waveguides in rare earth doped YAG," Opt. Lett., vol. 36, pp. 3395-3397, 2011.

[17] C. Grivas, C. Corbari, G. Brambilla, and P. G. Lagoudakis, "Tunable, continuous-wave Ti: Sapphire channel waveguide lasers written by femtosecond and picosecond laser pulses," Opt.Lett., vol. 37, no. 17, pp. 46304632, Sep. 2012

[18] J. Siebenmorgen, T. Calmano, K. Petermann, and G. Huber, "Highly efficient Yb: YAG channel waveguide laser written with a femtosecondlaser," Opt. Exp., vol. 18, no. 15, pp. 16035-16040, Jul. 2010.

[19] A. Benayas, W. F. Silva, C. Jacinto, E. Cantelar, J. Lamela, F. Jaque, J. R. Vázquez de Aldana, G. A. Torchia, L. Roso, A. A. Kaminskii, and D. Jaque, "Thermally resistant waveguides fabricated in Nd: YAG ceramics by crossing femtosecond damage filaments," Opt. Lett., vol. 35, no. 3, pp. 330-332, Feb. 2010.

[20] Y. Ren, J. R. Vázquez de Aldana, and F. Chen, "Near-infrared lasers and self-frequency-doubling in Nd: YCOB cladding waveguides," Opt. Exp., vol. 21, no. 9, pp. 11562-11567, May 2013.

[21] D. G. Lancaster, S. Gross, H. Ebendorff-Heidepriem, K. Kuan T. M. Monro, M. Ams, A. Fuerbach, and M. J. Withford, "Fifty percent internal slope efficiency femtosecond direct-written $\mathrm{Tm}^{3+}$ : ZBLAN waveguide laser," Opt. Lett., vol. 36, no. 9, pp. 1587-1589, May 2011.

[22] A. Okhrimchuk, V. Mezentsev, A. Shestakov, and I. Bennion, "Low loss depressed cladding waveguide inscribed in YAG: Nd single crystal by femtosecond laser pulses," Opt. Exp., vol. 20, no. 4, pp. 3832-3843, Feb. 2012.

[23] Y. Jia, J. R. Vázquez de Aldana, and F. Chen, "Efficient waveguide lasers in femtosecond laser inscribed double-cladding waveguides of Yb: YAG ceramics," Opt. Mater. Exp., vol. 3, no. 5, pp. 645-650, May 2013.

[24] Y. Tan, Q. Luan, F. Liu, F. Chen, and J. R. Vázquez de Aldana, "Qswitched pulse laser generation from double-cladding Nd: YAG ceramics waveguides," Opt. Exp., vol. 21, no. 10, pp. 18963-18968, Aug. 2013. 
[25] H. Liu, F. Chen, J. R. Vázquez de Aldana, and D. Jaque, "Femtosecondlaser inscribed double-cladding waveguides in Nd:YAG crystal: a promising prototype for integrated lasers," Opt. Lett., vol. 38, no. 17, pp. 3294 3297, Sep. 2013.

Yuechen Jia received the B.A. degree from Shandong University, Jinan, China, in 2010, where he is currently working toward the Ph.D. degree.

His current research interests include fabrication of optical waveguides in laser materials by using ion beam implantation and femtosecond laser inscription technique.

J. R. Vázquez de Aldana received the Bachelor of Science and Ph.D. degrees from the University of Salamanca, Salamanca, Spain. He is currently an Associate Professor of the Science Faculty, University of Salamanca.

His research interests include the interaction of intense femtosecond pulses with materials and its application to the fabrication of photonic devices. He is a member of the Laser Microprocessing Research Group, and is also a technical and scientific advisor of the Laser Facility at the University of Salamanca.

Qingming Lu was born in Shandong, China, in 1968. He received the B.A. degree from Shandong University, Jinan, China, in 1996.

He is currently a Senior Engineer in the School of Chemistry and Chemical Engineering, Shandong University, Jinan, China. His research interests include growth and characterization of optical crystals.
Daniel Jaque received the degree in physics from Sussex University, Brighton, U.K., in 1995, beginning his research in the fabrication and characterization of waveguides and photonic devices in nonlinear media by ion implantation. He received the Ph.D. degree in physics from the Universidad Autónoma de Madrid, Madrid, Spain, in 1999, where his research subject was the development of multifrequency solid-state lasers based on rare-earth-doped nonlinear crystals.

He moved to the Universidad Complutense de Madrid, Madrid, where he investigated the optical and superconducting properties of nanostructured thin films fabricated by electron beam lithography. In 2002, he joined the Material Science Department, Universidad Autónoma de Madrid. His current research interests include the fabrication of photonic devices by direct laser writing in a laser-gain medium and its characterization by means of fluorescence confocal microscopy by using laser ions as optical probes.
Feng Chen received the B.A. and Ph.D. degrees from Shandong Normal University and Shandong University, Jinan, China, in 1997 and 2002, respectively. In 2002, he was a Lecturer of physics at Shandong University, when he finished his doctoral dissertation on the ion-implanted optical waveguides and their applications in opto-electronics. From 2003 to 2005, he was engaged in research on discrete solitons in nonlinear periodic waveguide arrays or lattices as an Alexander von Humboldt Research Fellow in the Clausthal University of Technology, Germany. In 2004, he became an Associate Professor at Shandong University. In 2006, he became a Professor of physics at Shandong University, where he is currently with the School of Physics and State Key Laboratory of Crystal Materials. His current research interests include optical waveguides produced by energetic ion beams and ultrafast lasers, waveguide lasers, photorefractive solitons, and photonic crystals. He is currently a Senior Member of the Chinese Optical Society, a Senior Member of the Optical Society of America, a Fellow of the Institute of Physics, U.K., and the Director of the Society of Nuclear Physics, China. He also serves as an Associate Editor of Optical Engineering. 